\title{
What is always necessary throughout efforts to prevent and control COVID-19 and other infectious diseases? A physical containment strategy and public mobilization and management
}

\author{
Mingyu Luo ${ }^{\S}$, Jimin Sun ${ }^{\S}$, Zhenyu Gong* ${ }^{*}$ Zhen Wang* \\ Zhejiang Provincial Center for Disease Control and Prevention, Hangzhou, Zhejiang, China.
}

SUMMARY The COVID-19 pandemic continues to ravage the world. As many countries have entered the postpandemic period, current efforts to prevent and control COVID-19 have gradually been normalized in many countries. Although the focus is on vaccines to achieve herd immunity, conventional physical containment strategies should be reassessed as part of efforts to prevent and control infectious diseases. Continued respiratory protective measures such as social distancing and the wearing of masks have been extensively accepted by the public in most countries. A point worth noticing is that the activities of influenza and other respiratory diseases have decreased as these strategies have been implemented. Public mobilization and large-scale campaigns to promote health are also important to interrupting the transmission of pathogens. A good example can be found in the achievements of China's Patriotic Public Health Campaign. These practices underscore the importance of enhancing physical containment strategies and public mobilization and management, with support from the legal system, to respond to any potential emerging infectious diseases.

Keywords physical containment strategy, public mobilization, COVID-19, infectious disease

Though we are halfway through 2021, the COVID-19 pandemic continues to ravage the world (Figure 1). Many countries have implemented interventions to control the disease. Vaccines have been seriously considered to achieve herd immunity in order to control the COVID-19 pandemic (1), and different types of vaccines have proven effective (2). Under real-world conditions, vaccines still face many challenges to protecting the public against COVID-19 and other infectious diseases.

Without sufficient testing, vaccines may fail to fully eliminate or halt the spread of viral infections. Generally, the preparation phase takes too long to respond to outbreaks and other public health emergencies. Although COVID-19 vaccines have been developed rapidly in comparison to conventional vaccines and various approaches to evaluating vaccine efficacy have been devised, testing to evaluate safety and efficacy still takes time, and trials must assess the rate of protection afforded to multiple subpopulations, the reduction in the incidence or spread of infection, the severity of the resulting disease, and the duration of protection (from new variants) (4).

The availability of vaccines should be considered for them to be accepted and received by a large majority of the population. Availability includes sufficient storage, and especially in developing countries. Attention should also be paid to the willingness of the public to be vaccinated (5).

Vaccine breakthrough infections are expected. In one study, a small percentage of subjects (2 female subjects) who received the second dose of the NHT162b2 (Pfizer-BioNTech) or mRNA-1273 (Moderna) vaccine underwent viral testing weekly (6). Two female subjects were identified as vaccine breakthrough infections (infection was identified In Subject 119 days after the second dose and in Subject 236 days after that dose). According to surveillance by the US CDC, a total of 10,262 SARS-CoV-2 vaccine breakthrough infections had been reported from 46 US states and territories as of April 30, 2021. Those vaccine breakthrough infections included 2,725 asymptomatic patients (27\%), 995 patients who were hospitalized (10\%), and 160 patients who died (2\%) (7).

Although the focus is on vaccines, conventional physical containment strategies should be reassessed as part of efforts to prevent and control infectious diseases. Physical containment strategies can be divided into four levels based on the target and scale. Personal protections include the wearing masks, handwashing, and social distancing. Epidemiological measures include patient 


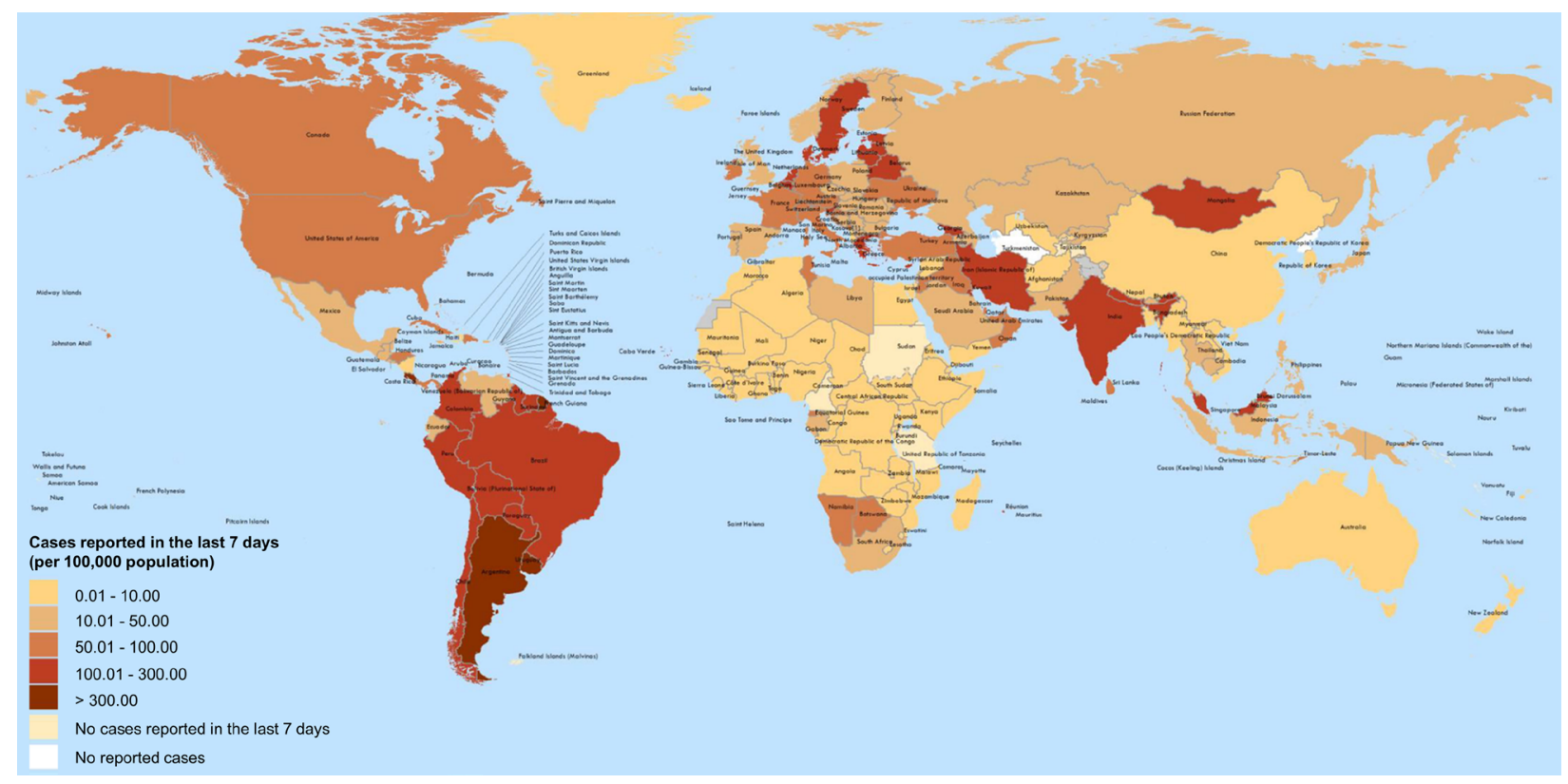

Figure 1. COVID-19 cases per 100,000 population reported by countries, territories, and areas, 17 May-23 May 2021. Figure is from the COVID-19 Weekly Epidemiological Update (3). Data presented are based on official laboratory-confirmed COVID-19 case and deaths reported to the WHO by country/territories/areas, largely based upon WHO case definitions and surveillance guidance.

isolation and contact tracing. Environmental measures include environmental disinfection and standard operating procedures in clinics and other key locations. Public mobilization and lockdown measures include cancelling of large gatherings, locking down of areas where a disease is likely to spread, and traffic control (8).

Due to continued concerns about controlling COVID-19, at a minimum, personal respiratory protection has been extensively accepted by the public in most countries. A point worth noticing is that the activities of influenza and other respiratory diseases have decreased while these strategies have been implemented. According to a report by the World Health Organization (WHO), seasonal influenza activity has decreased: influenza $\mathrm{A}(\mathrm{H} 1 \mathrm{~N} 1)$ pdm09, $\mathrm{A}(\mathrm{H} 3 \mathrm{~N} 2)$, and influenza $\mathrm{B}$ viruses circulated in very low numbers and the relative proportions of the viruses circulating varied among global reporting countries between September 2020 and January 2021 (9) (Figure 2). In Europe, only sporadic outbreaks of influenza A or B viruses were detected. In comparison to previous years, the number of specimens tested decreased $20 \%$.

Similar reductions in influenza and other respiratory infections have also been noted in Asian countries. According to the Tokyo Metropolitan Infectious Disease Surveillance Center, there have been fewer than 5 reported cases at each designated medical facility per week during the past year (week 36 of 2020 to week 36 of 2021) compared to a peak (64 cases/sentinel site) from week 36 of 2018 to week 36 of 2019 during the past 5 years (11). Based on a weekly database, a Japanese study clearly demonstrated that summer influenza disappeared in Okinawa Prefecture in 2020 (12).
Physical containment strategies that seek to interrupt the transmission of pathogens are effective at preventing and controlling COVID-19, influenza, and other infectious diseases.

As many countries have entered the post-pandemic period, the current efforts to prevent and control COVID-19 have gradually been normalized in many countries. However, the global public health system should aware that emerging infectious diseases (EIDs) require continuous vigilance. Since 2003, severe acute respiratory syndrome (SARS), Middle East respiratory syndrome (MERS), Zika virus disease, and other EIDs have rapidly developed and caused a series of serious public health incidents. Governments and public health agencies should emphasize research and practical implementation of conventional physical containment strategies. To prevent and control sexually transmitted diseases, personal behaviors such as condom use and remaining monogamous should be emphasized; to control the spread of infectious diseases of the digestive tract, environmental disinfection should be emphasized, such as preventing the contamination of drinking water and food, sanitary waste disposal, and eradication of flies. Many EIDs appear to be caused by zoonotic pathogens and involve interaction between humans and wildlife (13). To avoid infection with vectorborne diseases, entry into wildlife habitats should be limited, and wildlife should not be regularly consumed. Surveillance and elimination of vectors should also be emphasized. In Eastern China, an evaluation system with four indices was created: control of mosquito density, village administration, health education and public attitudes, and control of mosquito density via 

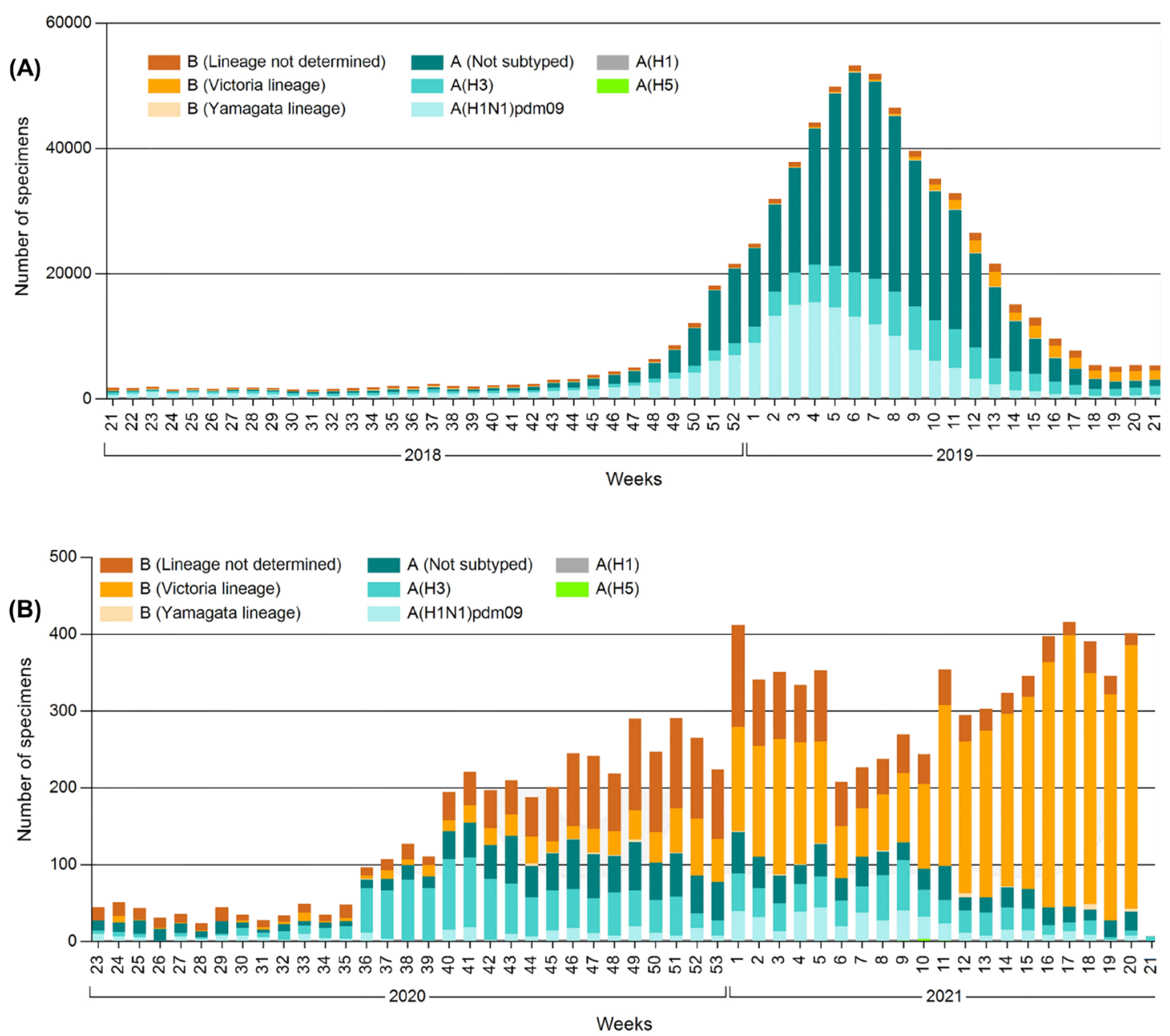

Figure 2. (A), Number of specimens positive for influenza by subtype from week 21 of 2018 to week 21 of 2019. (B), Number of specimens positive for influenza by subtype from week 21 of 2020 to week 21 of 2021. Figures are from the global influenza surveillance and response system (GISRS), WHO. Data presented are based on influenza laboratory surveillance information (10)

elimination of mosquito breeding grounds (14). After just one year, the mosquito density in a pilot village had decreased more than $90 \%(15)$. These measures demonstrate that physically separating vulnerable populations from potential pathogens is always an essential but economical and effective method of controlling infectious diseases. Public mobilization and large-scale campaigns to promote health are important to spreading those concepts and providing that health knowledge. The decades-long Patriotic Public Health Campaign in China is a good example of improving environmental health in urban and rural areas, and it has greatly improved public health. In Eastern China, local governments have combined efforts to prevent and control infectious diseases (such as vector-borne diseases) with rural revitalization in order to create a more comfortable and safer environment in rural villages (16). In July 2017, the WHO awarded the Chinese Government for its efforts and achievements during its Patriotic Public Health Campaign (17).

To ensure the effectiveness of prevention and control measures, a complete public health system should be envisioned and implemented. This means not just facilities but also science-based prevention and control efforts. Legal regulations ensure that the public health system functions legally and effectively. The World Health Assembly (WHA) adopted the new International Health Regulations (IHR) on May 23, 2005 , and the purpose of the new IHR is "to prevent, protect against, control, and provide a public health response to the international spread of disease in ways that are commensurate with and restricted to public health risks and which avoid unnecessary interference with international traffic and trade" (18). Moreover, legal regulations help improve the public health system's response. A more resilient and responsive public health system needs support from the legal system. A study in the Netherlands retrospectively compared the effects of legislative changes and increased awareness on the timeliness of notification (of local public health authorities) and reporting (to national public health authorities) during 10 outbreaks (19). Results indicated that the average delay in notification decreased from 1.4 to 0.4 day once the changes took effect (six diseases; $p<$ 0.05 ), and the delay in reporting mainly decreased right after the changes took effect (from 0.5 to 0.1 day, six 
diseases; $p<0.05$ ).

The role of legislation should be emphasized to enhance the WHO's emergency response to acute infectious diseases or public health emergencies. This means that the previous IHR (2005) needs to be modified. The legal status of measures to prevent and control infectious diseases should be enhanced, and new IHR (2022) may be adopted to enhance public mobilization and management.

As globalization continues, the threat of infectious diseases is ever-present. Governments should always prepare to implement a containment strategy and mobilize the public. The public health system and related support from the legal system should both be seriously considered. During the formulation of physical containment strategies, their advantages and disadvantages should be fully evaluated to identify the most effective strategies to protect public health, with due consideration to personal freedom of movement. Lastly, positive aspects can be maximized to promote both global public health and economic development.

Funding: This work was granted by the National Critical Project for Science and Technology on Infectious Diseases of P. R. China (No. 2017ZX10303404).

Conflict of Interest: The authors have no conflicts of interest to disclose.

\section{References}

1. Chen J, Lu H. New challenges to fighting COVID-19: Virus variants, potential vaccines, and development of antivirals. Biosci Trends. 2021;15:126-128.

2. Kashte S, Gulbake A, El-Amin SF, Gupta A. COVID-19 vaccines: Rapid development, implications, challenges and future prospects. Hum Cell. 2021; 34:711-733.

3. WHO. COVID-19 Weekly Epidemiological Update. World Health Organization. 2021; https://www.who. int/docs/default-source/coronaviruse/situation-reports/ weekly_epidemiological_update_22.pdf (accessed May 31, 2021).

4. Hodgson SH, Mansatta K, Mallett G, Harris V, Emary KRW, Pollard AJ. What defines an efficacious COVID-19 vaccine? A review of the challenges assessing the clinical efficacy of vaccines against SARS-CoV-2. Lancet Infect Dis. 2021; 21:e26-e35.

5. Pogue K, Jensen JL, Stancil CK, et al. Influences on attitudes regarding potential COVID-19 vaccination in the United States. Vaccines. 2020; 8:1-14.

6. Hacisuleyman E, Hale C, Saito Y, Blachere NE, Bergh M, Conlon EG, Schaefer-Babajew DJ, DaSilva J, Muecksch F, Gaebler C, Lifton R, Nussenzweig MC, Hatziioannou T, Bieniasz PD, Darnell RB. Vaccine Breakthrough Infections with SARS-CoV-2 Variants. N Engl J Med. 2021; NEJMoa2105000. doi: 10.1056/NEJMoa2105000.

7. CDC COVID-19 VBCI Team. COVID-19 Vaccine Breakthrough Infections Reported to CDC - United States, January 1-April 30, 2021. Morb Mortal Wkly Rep. 2021; 70:792-793.
8. Chen W, Wang Q, Li YQ, et al. Early containment strategies and core measures for prevention and control of novel coronavirus pneumonia in China. Zhonghua $\mathrm{Yu}$ Fang Yi Xue Za Zhi. 2020; 54:239-244. (in Chinese)

9. World Health Organization. Weekly Epidemiological Record. 2021; 96:77-78. https://reliefweb.int/sites/ reliefweb.int/files/resources/WER9611-eng-fre.pdf (accessed May 31, 2021).

10. World Health Organization. Influenza virus detections, Global circulation - optional time period selection. https:// apps.who.int/flumart/Default? Report $N o=10$ (accessed May 31, 2021).

11. Tokyo Metropolitan Infectious Disease Surveillance Center. The prevalence of influenza from 2020-2021. http://idsc.tokyo-eiken.go.jp/diseases/flu/flu/ (accessed May 31, 2021). (in Japanese)

12. Sunagawa $\mathrm{S}$, Iha Y, Kinjo T, Nakamura K, Fujita J. Disappearance of summer influenza in the Okinawa prefecture during the severe acute respiratory syndrome coronavirus 2 (SARS-CoV-2) pandemic. Respir Investig. 2021; 59:149-152.

13. Daszak P, Cunningham AA, Hyatt AD. Emerging infectious diseases of wildlife - Threats to biodiversity and human health. Science. 2000; 287:443-449.

14. Chen EF, Guo S, Huang WZ, Sun JM, Gong ZY. The mosquito control and evaluation system of "mosquitofree village" construction in rural areas. Prev Med. 2019; 31:217-220. (in Chinese)

15. Wu HZ, Liu Y, Ling F, Sun JM, Gong ZY, Hou J, Chen EF. Evaluation on construction of "mosquito-free village" in Pujiang county, Zhejiang, China. Chin J Vetor Biol Control. 2018; 29:283-286. (in Chinese)

16. Gong ZY, Liu QM, Wang JN, Hou J. New connotations for patriotic health campaign with Chinese characteristics in new era - Exploration of vector prevention and control pattern in Zhejiang. Dis Surveill. 2021; 36:312-317. (in Chinese)

17. China NHC of PR of. WHO Rewarded China Government's Achievements during 65 Years of Patriotic Sanitation Campaign.; 2017. http://www.nhc.gov.cn/wjw/ ttyw/201707/b7bc50544d0b4d6d9efd175fad663754.shtml (accessed May 31, 2021). (in Chinese)

18. Fidler DP, Gostin LO. The new international health regulations: An historic development for international law and public health. J Law Med Ethics. 2006; 34:85-94.

19. Swaan CM, Wong A, Marinović AB, Kretzschmar MEE, Van Steenbergen JE. Timeliness of infectious disease reporting, the Netherlands, 2003 to 2017: Law change reduced reporting delay, disease identification delay is next. Eurosurveillance. Euro Surveill. 2019; 24:1900237.

Received May 31, 2021; Revised June 11, 2021; Accepted June 13, 2021.

${ }^{\S}$ These authors contributed equally to this work.

*Address correspondence to:

Zhenyu Gong and Zhen Wang, Zhejiang Provincial Center for Disease Control and Prevention, No. 3399 Binsheng Road, Binjiang District, City of Hangzhou 310051, Zhejiang, China. E-mail: zhygong@cdc.zj.cn (Gong ZY), wangzhen@cdc.zj.cn (Wang Z)

Released online in J-STAGE as advance publication June 16, 2021. 